\title{
SIVEQ: an Integrated System for the Valorization of Surplus Food
}

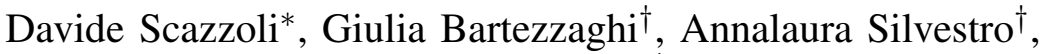 \\ Maurizio Magarini*, Marco Melacini ${ }^{\dagger}$, Giacomo Verticale* \\ *Dipartimento di Elettronica, Informazione e Bioingegneria, Politecnico di Milano, Italy \\ Email:\{davide.scazzoli,maurizio.magarini,giacomo.verticale,annalaura.silvestro,marco.melacini\}@polimi.it \\ ${ }^{\dagger}$ Dipartimento di Ingegneria Gestionale, Politecnico di Milano, Italy \\ Email: giulia.bartezzagh@ osservatori.net
}

\begin{abstract}
Food waste is one of the key challenges of the agri-food sector: one third of the global food production is wasted yearly, while paradoxically 815 million people do not have access to sufficient and nutritious food. Food waste represents an economic loss for the agri-food supply chain and the whole society and significantly contributes to the GHG emissions. In Italy up to 5.1 million tons of food is wasted: nearly half of it is generated by agri-food supply chain actors. Retailers contribute to the $14 \%$ of the overall food waste produced and the main cause relies on products reaching the expiration date. Over the last years retailers have increasingly taken action in order to recover surplus food, encouraged by positive changes in the regulatory environment and the increasing relevance of Corporate Social Responsibility policies adopted by companies. Food donations have been increasing, but in many cases the surplus food redistribution process to food-aid organizations is still occasional and not formalized, leaving space for efficiency improvement. Surplus food close to expiration date, if not properly and timely handled, inevitably turns into waste. In this paper we introduce SIVEQ: a systematic solution which relies on novel technologies such as IoT and big data analytics to tackle this issue. Our system represents an added value to all actors involved, not only for NPOs who collect and redistribute surplus food.
\end{abstract}

\section{INTRODUCTION}

Food waste is becoming an increasingly important issue at both micro and macro economic scales. Furthermore the greenhouse gas (GHG) emissions from food production, consumption, as well as from its final disposal contribute a significant portion of the total world emissions. Estimates show that GHG emissions due to food waste may rise at astonishing rates of $1.9-2.5 \mathrm{Gt}$ of $C O 2_{e q} / y r$ [1]. Depletion of natural resources and pollution are the most prominent environmental impacts associated with food waste.

Food waste represents an economic burden for all actors involved in the food production, processing and distribution industry as well as for the final consumer. The costs not only include the losses from the unsold food products but also the cost associated with their disposal once they become food waste. Given the ever increasing world population, the need to provide sustainable and efficient food management that minimizes food waste becomes of critical importance for a sustainable economy [2].

Many options have been investigated for reducing food waste, especially when it is avoidable, ranging from public sensitization to the environmental impact of food waste and improved consumption patterns, to improved labeling, better demand planning and redistribution strategies for super markets [3], [4].

The Food Waste Hierarchy (FWH) framework, shown in Figure 1, helps companies set clear priorities for action to reduce food waste [5]. The overall objective is that surplus food always finds a destination of use, from human consumption to recovery for energy production, leaving the disposal in landfill as the last option. According to the $\mathrm{FWH}$, the first priority for companies and other actors committed to food waste reduction is the prevention of surplus food generation and, once the latter is generated, its reuse or redistribution for human consumption, preventing it from turning into "social" waste. Here the activities of surplus food recovery and redistribution in favour of people 


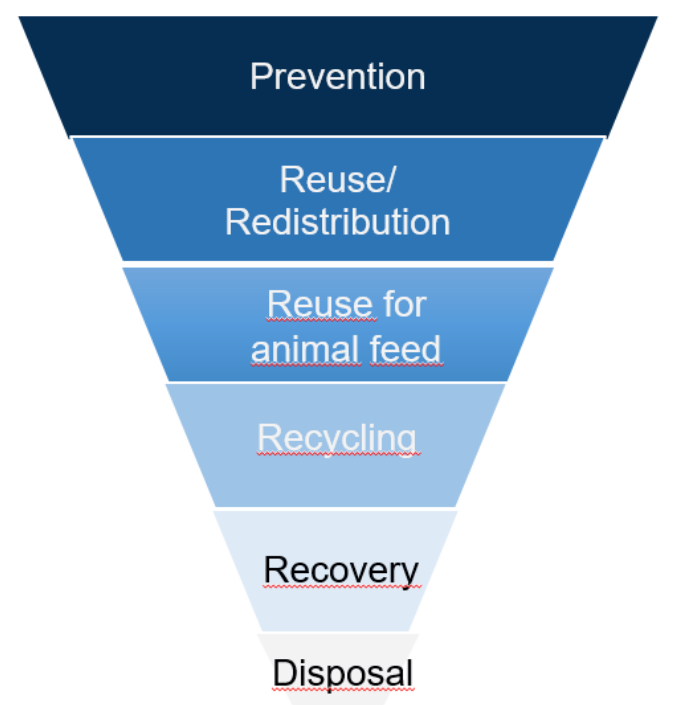

Fig. 1. The Food Waste Hierarchy (FWH) represents the order of desirability for different types of approaches to reducing food waste [5].

in need take place, thanks to the joint effort of companies and food-aid organizations. Companies have already begun such practices as they represent not only positive publicity but also have clear cost saving benefits [6].

In this paper we present a system which focuses primarily on the second option: redistribution of surplus food before it becomes unfit for human consumption. The objective of the platform Sistema Integrato per la Valorizzazione delle Eccedenze Alimentari nel Quartiere (SIVEQ, "Integrated system for the valorization of surplus food in the district" in English) is to enable information sharing between all the actors involved in the redistribution of surplus food, from the food distributors that donate excess food to the logistic and finally the beneficiary, usually Nonprofit Organizations (NPO), in order to minimize food waste. The idea of redistributing surplus food for social purposes is not novel as it has been adopted before, however it could benefit from a more systematic approach that integrates novel technologies such as IoT and Big Data analysis [7]. Our system enables the best practices highlighted by previous case studies to be integrated into business processes with minimal human resource costs. This work was entirely funded from the proposal sharing the same name (SIVEQ, Integrated system for the valorization of

\begin{tabular}{|c|r|r|r|}
\hline Stage & $\begin{array}{c}\text { Surplus food } \\
\text { [tons] }\end{array}$ & $\begin{array}{c}\text { Waste } \\
\text { [tons] }\end{array}$ & $\begin{array}{c}\text { Total } \\
\text { incidence }\end{array}$ \\
\hline Primary & 2.045 .000 & 1.755 .000 & $34 \%$ \\
\hline Transformation & 175.000 & 75.000 & $1 \%$ \\
\hline Distribution & 755.000 & 690.000 & $14 \%$ \\
\hline Food services & 210.000 & 185.000 & $4 \%$ \\
\hline Consumption & 2.405 .000 & 2.405 .000 & $47 \%$ \\
\hline
\end{tabular}

Table I: Food surplus and food waste quantification for Italy [2].

surplus food in the neighborhood), winner of the POLISOCIAL Award 2017. This award aims to fosters scientific research with a high social impact and is funded with the tax-return funds donated to Politecnico di Milano.

The rest of the paper is organized as follows: Sec. II analyzes the problem of surplus food and possible logistics approaches. Sec. III introduces our system architecture and explores its components. Sec. IV covers the challenges faced during the development of the project and finally sec. V concludes the paper.

\section{PROBlem ANALYSis}

The main focus of this project is on the distribution and food services sectors where, as shown in table I, a notable portion of food waste is created[2], [8]. In most cases, surplus food is generated due to a misalignment between demand and supply, which in turn derives from forecasting errors or market imperfections. For retailers the main cause of food waste relies on products reaching the internal sellby-date. Due to such commercial policies a certain amount of surplus food will always be produced and it varies according to unpredictable market variation and customer trends. It is therefore imperative that this surplus is transformed not into waste but into a resource to be used for social purposes.

The first choice for the redistribution of surplus food is whether to adopt a centralized or distributed approach. In fig. 2 the main differences between the two distribution methods are shown. Current approaches to surplus redistribution rely on distributed approaches as they reduce the complexity and costs on the parts of the donors.[9] This approach is the simplest and most unorganized one as it naturally emerged as the actors involved spontaneously come together to accomplish their respective goals. In contrast to this, a centralized approach requires 


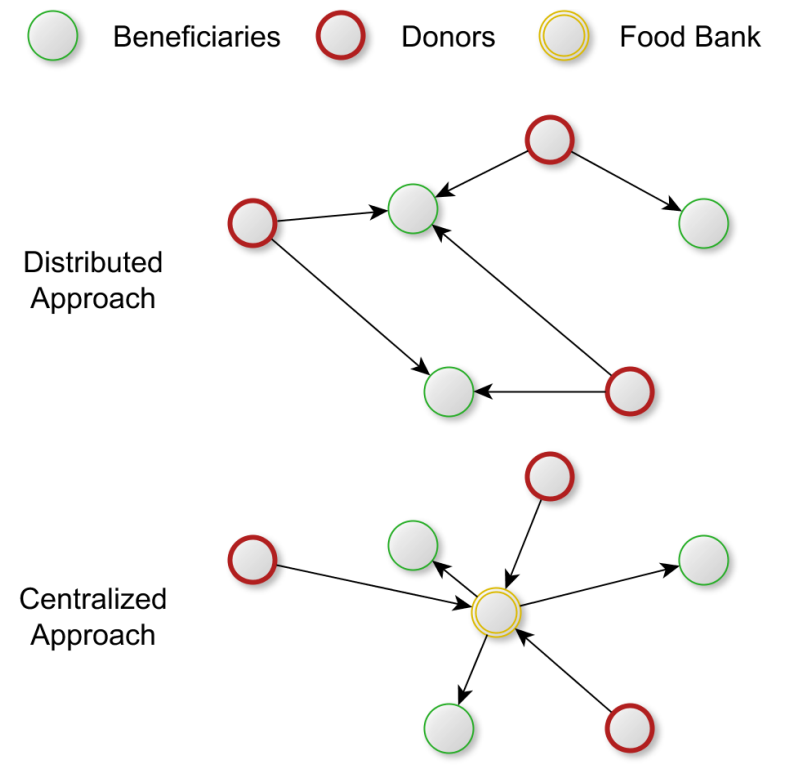

Fig. 2. illustration of distributed capillary and centralized relying on food banks approaches to surplus food redistribution

more investment, an intermediary food bank entity is also required further increasing costs. The centralized approach brings several benefits that offset its higher costs:

- A single donor is able to reach a higher number of beneficiaries thus further reducing waste and saving more money on waste disposal.

- The collection of all food donation in a single place facilitates quality control procedures.

- Having only one logistical intermediary allows for the optimization of collection and distribution itineraries using proven technologies aided by big data analytics.[10]

This in turn allows us to adopt good practices for the management of surplus food which have been identified in the following:[2]

- Proactive management with periodic donations

- formal coordination with all actors involved

- systemic approach for all products involved

- periodic measurements

\section{System ARChitecture}

The aim of the SIVEQ platform is to implement a data acquisition procedure centered on an handheld device, a backend database for storing data about surplus food products in supermarkets or other distributors and a web interface for letting NPOs interact with this data. The objective is providing collection of information on quantity, status and the expiration date through the use of devices very similar to the barcode scanners currently used in stores. Thus, when surpluses are removed from trade, their availability is immediately notified to NPOs and the other actors involved, such as intermediary food banks.

The main architecture of the system is shown in figure 3, the crucial innovation parts are the following:

- A donor application which is able to quickly collect and upload all required information on surplus food as soon as they are taken off the shelf.

- A cloud-based database for storing all this information as well as analytic or machine learning algorithm for optimizing the logistics and forecasting possible future surplus.

- A web based secure interface where the NPOs can privately browse the available surplus information and request the delivery of, or collect unclaimed surplus food.

Another function that can be integrated in the system, depending on the hardware used, is to take pictures of the product to check the state of the packaging. By means of a software installed on the devices supplied to the staff, the information will be automatically uploaded to the internet using the existing networks (Wi-Fi, LTE) or new technologies (5G, NB-IoT). Our focus is on wireless technologies as they are best integrated in current processes and leave the operators unhindered. The information uploaded is sensitive data, as it inherently highlights inefficiencies in the donor's management, therefore proper security protocols and privacy must be considered, furthermore, a control interface will let the donor manage which information should be shared or preemptively decide which NPO should be the beneficiary. Inside the Cloud, information from the various points of sale will be collected and analyzed in order to meet the food requirements charged by the NPOs.

After performing several interviews with distributors and ONPs which will be directly involved in the deployment of the first experimental platform, we have identified problems in terms of holes in the distribution coverage of ONPs and difficulty of communication between distributors and ONPs. Given the data collected, it will be possible to 


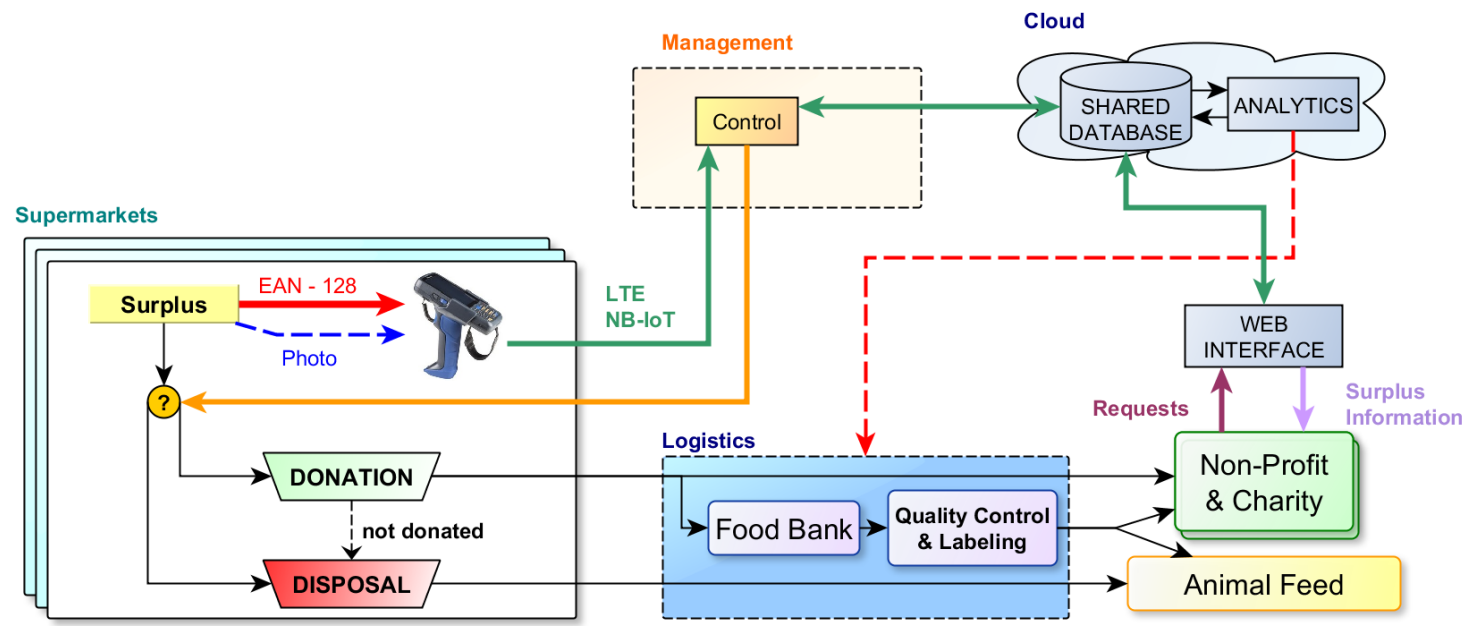

Fig. 3. SIVEQ System architecture

employ it for eliminating such problems, optimizing the logistics and providing opportunities for implementing Big Data Business Analysis (BDBA)[11]. The NPO will be able to view in real time the status of the surpluses assigned to them and better prepare to receive them, transform them and eventually integrate them with purchases. The WEB interface will have the role of facilitating this process for the NPOs and will therefore be a web page or an application.

\section{A. Donor application}

When the application is launched, the initial interface consists of a view where the user logs in, the activity flow is shown in fig.4. After log in the user is greeted with a view where they can choose either to check the list of items that are already added in the database, add another product or remove a product. This GUI is meant for the donors which will scan the products they wish to make available for donation, hence, its design has been streamlined to reduce the effort required by the user during normal operation as much as possible. The products thus uploaded will have color bar indicators that will make it easier to know which items are expired or valid. There will also be an option of removal of items once they expire from the list as well as the database. In case the user clicks the button add, they will be taken to the "add Product" activity where the barcode scanner is used to get the product GTIN, expiry date and other information. Such information will be then uploaded to our webserver as a JSON object, in

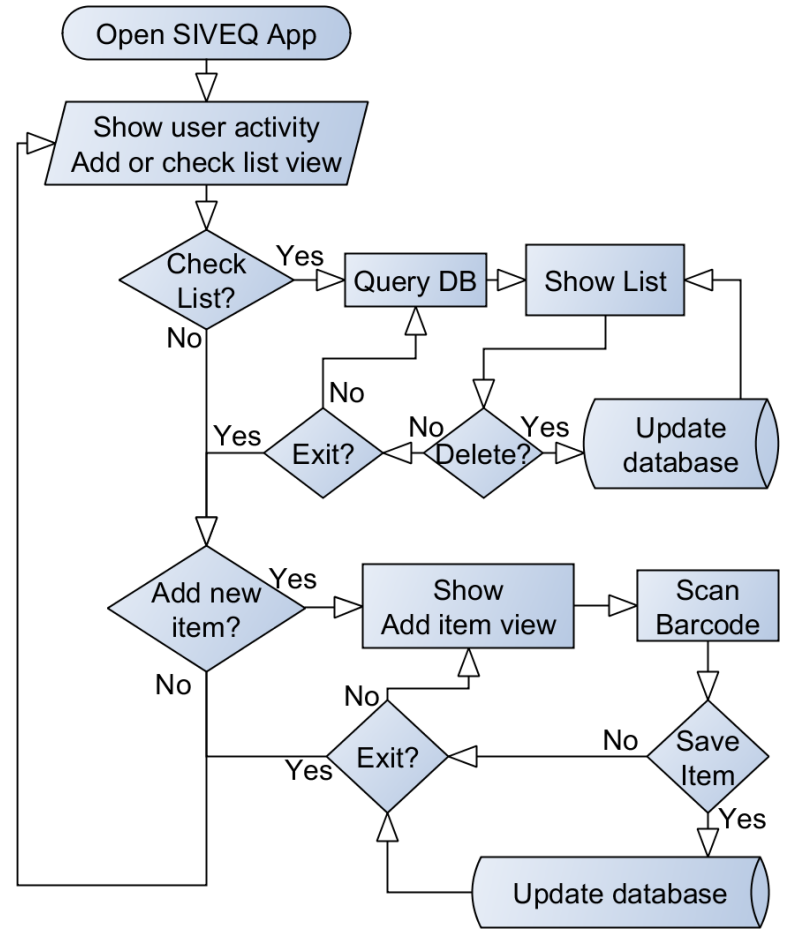

Fig. 4. Donor application flowchart, after scanning the barcode the product information is automatically acquired and can be manually edited.

order to be stored in the database, as will be shown in sec. III-C. In case the scanning module activity is not able to associate a product Name/description or expiry date to the scanned barcode a manual input option is provided. For the acquisition of the expiry date, in the case it is missing from the barcode, there are several manual options. From a quick press of a button to indicate 1,2 or $3+$ days 


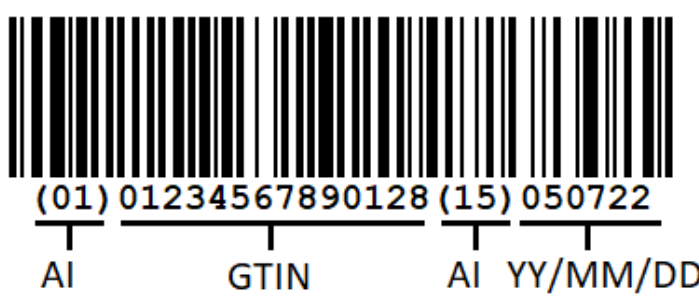

Fig. 5. GS1 Barcode example containing GTIN, with AI 01 and best before date, with AI 15

to expiry to a manual insertion that opens up a calendar to specify the date or even an interface to an Optical Character Recognition (OCR) software to attempt reading from a product image. In our particular implementation we used Tesseract as the choice OCR.[12]

\section{B. Usage of barcodes and their standards}

This system relies on well established practices of barcode scanning for acquiring most of the essential information. The GTIN (Global Trade Item Number) barcode system which has been globally standardized and implemented, is reviewed as a mandatory food-labeling regulation. The integration of GS1 bar-code system for food-traceability is a crucial factor to expand its function in the foodrelated industrial areas.[13] Because of this we consider a system where all the information can be gathered from the barcode present on the product, as shown in fig.5.

The barcode can be acquired by means of PDA equipped with dedicated barcode scanners. Such hardware is, however, usually rather expensive. A cheaper alternative is to use a generic smartphone's camera to acquire the barcode from a picture using a software library. In our project both options are considered, for the hardware scanner we used a HighTon MT6737, while for the software scanner we used the ZXing library.[14]

\section{Shared Database}

For our system, the main challenge in designing the database was to find the right structure. We did not have any access to a predefined structure, and the scope of the project included many heterogeneous data sources, from supermarkets to canteens. We opted for a NoSQL approach as it allowed superior scalability, and the ease of implementation or ongoing database modification that our project requires. For our particular project

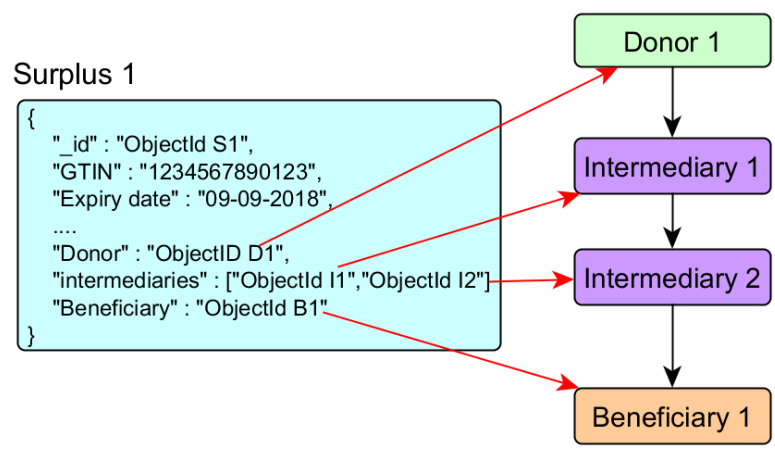

Fig. 6. Surplus document entry example with links to donor, beneficiaries and two logistical intermediaries

we used MongoDB [15] which is an open source NoSQL database.

The database consists in two collections, one for tracking surplus and one for tracking entities such as donors, beneficiaries or food banks. Inside these collections each surplus instance and each entity are stored as documents with unique Object Id's. This ObjectId is a 12 byte string automatically generated by the database for each document inserted, which is guaranteed to be unique by the database software. To each surplus entry, the Object Id of source donor, relevant intermediaries and destination beneficiary will be linked using MongoDB's citation mechanism, by doing this it will be immediate to query the relevant data such as how much surplus has been donated by a particular entity. This process will allow us to keep track of surplus at every stage, from creation to final consumption by NPOs.

\section{Challenges}

While the idea behind the project is not very complicated, during the development of our initial prototypes for the experimentation phase we faced several challenges:

- Expiry date acquisition: Normally, in the chain of distribution, the expiry date is kept coded inside the barcode. However, during retail sale, products with different expiry dates may end up mixed together and the barcode for retail sale may not contain the expiration date as it is instead printed on the product. It is therefore necessary to re-acquire this information when the product is donated. The first considered approach relied on the usage of OCR software to extract the expiry date from 


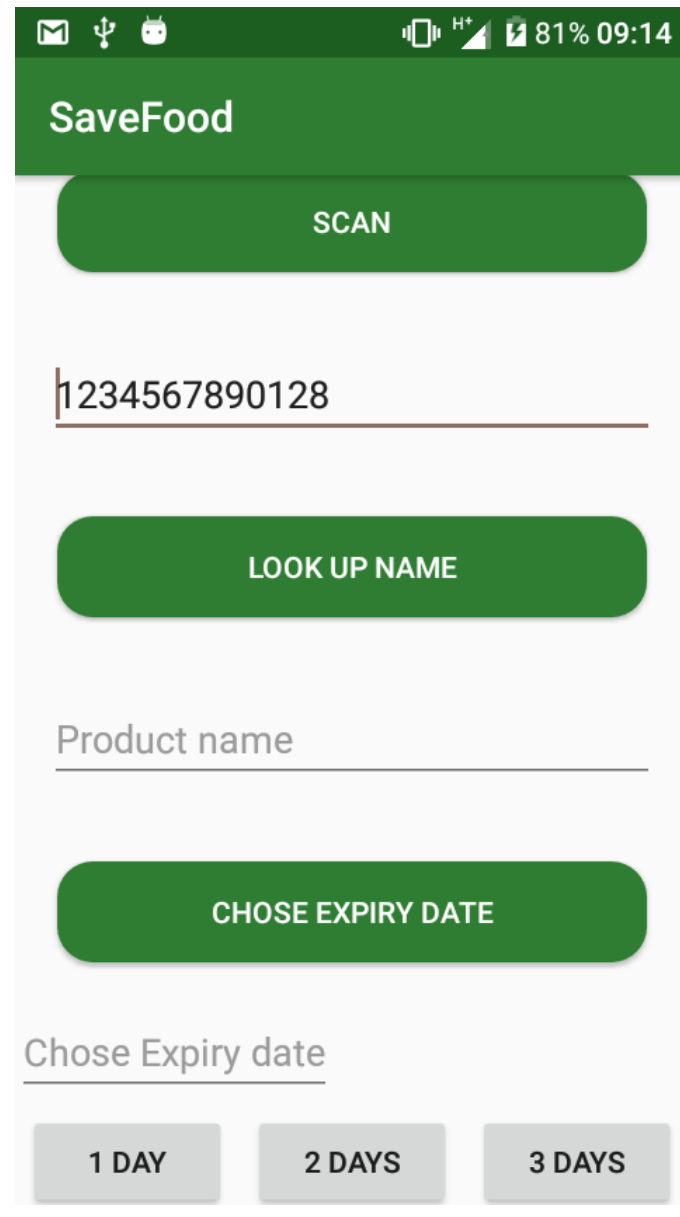

Fig. 7. Screenshot of the android app developed for the upload of product information. Within the same activity the user can scan the barcode, lookup product description from scanned barcode and assign expiry date with the click of a button.

an image of the product. This approach is the best at minimizing user input, however, despite using state-of-the-art software such as google tesseract[12], we were unable to obtain reliable results.

A second approach considers a minimalist user interface that shows few options for expiry date, such as "1 day", "2 days" and "3 days", as shown in Figure 7, where the user selects by pressing just a button. Thus getting user input in the fastest way possible, this approach is generally more reliable but is still prone to user error.

- Database size constraints and access restrictions: The data uploaded to the cloud database pertains to very large volumes of products for large supermarkets, thus efficiently stor- ing and indexing such data is a fundamental requirement. Furthermore, this data represents inefficiencies on the side of donors, thus it is sensible data that must not be shared without explicit consent from the donor. Options for anonymous donations which can be tracked only by law enforcement or government must be provided. All beneficiaries must be properly registered and access to data must be duly regulated.

- Legacy standard integration: Another challenge we faced was the integration of old standards or non-standard approaches due to currently widespread malpractice. One example is the usage of fictitious GTIN numbers for identifying local market products which invalidates the reliability of global product databases which adhere to the GS1 standard. An integration of the information related to this fictitious GTIN must be made in order to make sense of the scanned barcode, in our project we allow the possibility of uploading such information in an excel sheet format.

- Business Management issues: There is still much resistance to the adoption of good practices as the added benefit may not be perceived as sufficient to justify the investment in changing consolidated management practices.

The shift towards a more centralized approach based on food banks, which our system would rely on, is also facing challenges as the actors involved are often in competition with one another. This is especially true for the food donors, which in turn drives them to great wariness when relying on food banks that have ties with direct competitors.

\section{CONCLUSION}

In this paper we have presented a novel approach to the exploitation of surplus food for social reuse by Non-Profit Organizations based on IoT technologies which enable the adoption of Big Data Analytics. The system presents benefits for all actors involved and allows the creation of new market opportunities. With the adoption of the system outlined in this paper a reduction of the food waste in the distribution and catering sectors is expected, verification of this assumption via experimentation has already begun and further works in this project will include feedback from the distributors and 
Nonprofit Organizations that have taken part in it as well as experimental results. Other future works will explore how other emerging technologies, such as machine learning, that can help predict market trends and further reduce food waste and optimize the process and logistics.

\section{REFERENCES}

[1] C. Hic, P. Pradhan, D. Rybski, and J. P. Kropp, "Food surplus and its climate burdens," Environmental science \& technology, vol. 50, no. 8, pp. 4269-4277, 2016.

[2] P. Garrone, M. Melacini, and A. Perego, "Surplus food management against food waste," Politecnico di Milano, Milan, Italy, 2015.

[3] S. Baglioni, B. De Pieri, and T. Tallarico, "Surplus food recovery and food aid: The pivotal role of non-profit organisations. insights from italy and germany," VOLUNTAS: International Journal of Voluntary and Nonprofit Organizations, vol. 28, no. 5, pp. 2032-2052, 2017.

[4] O. Forcada, S. Sert, V. Soldevila, and F. Vidali, "Surplus food redistribution: Best practices from european countries," in Foodsaving in Europe, pp. 69-95, Springer, 2017.

[5] E. Papargyropoulou, R. Lozano, J. K. Steinberger, N. Wright, and Z. bin Ujang, "The food waste hierarchy as a framework for the management of food surplus and food waste," Journal of Cleaner Production, vol. 76, pp. 106-115, 2014.

[6] S. Sert, P. Garrone, M. Melacini, and A. Perego, "Corporate food donations: altruism, strategy or cost saving?," British Food Journal, vol. 120, no. 7, pp. 1628-1642, 2018.

[7] M. Marjani, F. Nasaruddin, A. Gani, A. Karim, I. A. T. Hashem, A. Siddiqa, and I. Yaqoob, "Big iot data analytics: architecture, opportunities, and open research challenges," IEEE Access, vol. 5, pp. 5247-5261, 2017.

[8] F. HLPE, "Food losses and waste in the context of sustainable food systems," A Report by the High Level Panel of Experts on Food Security and Nutrition of the Committee on World Food Security. Available online: http://www.fao.org/3/a-i3901e.pdf (accessed on 2 October 2017), 2014.

[9] S. Sert, P. Garrone, M. Melacini, and A. Perego, "Surplus food redistribution for social purposes: The case of coop lombardia," in Organizing Supply Chain Processes for Sustainable Innovation in the Agri-Food Industry, pp. 153-173, Emerald Group Publishing Limited, 2016.

[10] H. Shahrokni, B. Van der Heijde, D. Lazarevic, and N. Brandt, "Big data gis analytics towards efficient waste management in stockholm.," in ICT4S, 2014.

[11] G. Wang, A. Gunasekaran, E. W. Ngai, and T. Papadopoulos, "Big data analytics in logistics and supply chain management: Certain investigations for research and applications," International Journal of Production Economics, vol. 176, pp. 98-110, 2016.

[12] T. O. S. O. Engine, "Google tesseract github," Retrived from https://github. com/tesseract-ocr/tesseract.

[13] U. C. Council, "Gs1 databar family," Available from: Lawrenceville NJ: Uniform Code Council https://www. gs1. org/barcodes/databar, vol. 6, 2017.
[14] "Zxing, open source library to read $1 \mathrm{~d} / 2 \mathrm{~d}$ barcodes." Available at: https://github.com/zxing/zxing, Accessed: 15/01/2019.

[15] "Mongodb." Open Source Document Database, https://www.mongodb.com/. Accessed: 17/01/2019. 